NASA Technical Memórandum 88358

\title{
Application of Digital Holographic Interferometry to Pressure Measurements of Symmetric, Supercritical, and Circulation- Control Airfoils in Transonic Flow Fields
}

Francisco J .Torres

(AASA-TM-8835E) EEEIICATICA CF LIGITAL

ECLOG FAEHIC IATEEFEFCAETEY IC EEESSURE

MEASUREMENTS CF SYUMETEIC, SCEFHCEITICAL AND

CIRCULATICN-CCNIECL AIFFCIIS IS TEANSONIC

FLOW EIELDS (NASA) $23 p$ avail: ATS HC

June 1987
$N 87-24 \in \varepsilon 1$

Unclas

G3/35 0082703

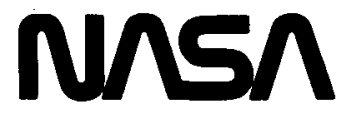

National Aeronautics and

Space Administration 


\section{Application of Digital Holographic Interferometry to Pressure Measurements of Symmetric, Supercritical, and Circulation- Control Airfoils in Transonic Flow Fields}

Francisco J. Torres, Ames Research Center, Moffett Field, California

June 1987

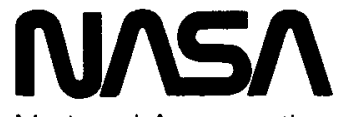

National Aeronautics and

Space Administration 


$\begin{array}{ll}C_{y} & \text { pressure coefficient } \\ K_{G-D} & \text { Gladstone-Dale constant } \\ L & \text { test section width and height } \\ L_{e f f} & \text { effective optical path length } \\ M_{\infty}, \mathrm{MACH} & \text { freestream Mach number } \\ N & \text { fringe order number } \\ X / C & \text { nondimensional chord length } \\ \alpha, A & \text { angle of attack } \\ \kappa & \text { ratio of specific heats } \\ \lambda & \text { laser wavelength } \\ n_{0} & \text { ambient (or reference) refractive index } \\ \rho_{r \in f} & \text { reference fluid density } \\ \rho_{x, y} & \text { fluid density at any point } \mathrm{x}, \mathrm{y} \\ \rho_{\infty} & \text { freestream fluid density }\end{array}$




\section{SUMMARY}

Six airfoil interferograms were evaluated using a semiautomatic image-processor system which digitizes, segments, and extracts the fringe coordinates along the airfoil surface. The resulting "fringe order function" was converted into density and pressure distributions and a comparison was made with pressure transducer data at the same wind tunnel test conditions. Three airfoil shapes were used in the evaluation to test the capabilities of the image processor with a variety of flows. Symmetric, supercritical, and circulationcontrol airfoil interferograms provided fringe patterns with shocks, separated flows, and high-pressure regions for evaluation. Regions along the airfoil with very clear fringe patterns yielded results within $1 \%$ of transducer measurements, while poorer quality regions, particularly near the leading and trailing edges, yielded results that were not as good.

\section{INTRODUCTION}

Holographic interferometric techniques have been widely used in recent years for flow visualization and quantitative measurements of flow properties. The typical method of evaluating interferograms is to read the fringe numbers and their positions manually or by tracing the fringe lines by hand with the help of a tracking device such as a graphic tablet. This process tends to be a very time-consuming and inaccurate procedure when large numbers of interferograms have to be evaluated. The use of an automatic fringe-reading procedure would therefore speed up the process, enhance the evaluation, and make the interferometric technique a much more powerful measurement tool.

In this paper, a digital interferogram analysis method developed by Becker (unpublished paper) on an image-processing system has been used to automatically digitize and evaluate the interferograms to obtain surface data for a symmetric, a supercritical, and a circulation-control airfoil. The evaluation of interferograms using computer-aided methods can be subdivided into the following steps 1) digitization and image enhancement, 2) fringe segmentation and fringe-coordinate extraction, 3) merging of fringe fields obtained from several magnified views, 4) fringe numbering and correction of fringe disconnections, 5) coordinate transformations, 6) interpolation and extrapolation of fringe-number functions, 7) conversion of fringe numbers into interesting flow properties, and 8) reconstruction of the flow field (ref. 1). Different computer programs were developed to perform these steps and the digital interferogram analysis method uses these modules interactively to accomplish the required evaluation.

\section{EXPERIMENTAL APPARATUS AND TECHNIQUES}

\section{Image-Processing System}

A De Anza image-processing system connected to a VAX 11/780 host computer provides the main hardware necessary for digitizing and evaluating interferograms (fig. 1). The resolution of the system is $512 \times 512$ pixels with an intensity range of 256 ( 8 bit). 
Currently, it is equipped with two of a possible four memory planes for image storage and with a graphic and an alphanumeric overlay. A frame-grabbing unit can digitize a frame of a video signal in real time from a black and white video camera which is connected to this input channel. The system has an arithmetic logic unit (ALU) with which real-time addition, subtraction, or comparison of one or more image planes may be made (ref. 1) The contents of each memory plane may be routed through lookup tables before being input to the ALU and can be shown on a color display. A joystick control device is used for interactive input. It controls two cursors, which may be used in a number of operating modes. A color print system serves as a hardcopy device for the color monitor. The user can automatically digitize and evaluate interferograms by interactively using the hardware units of the image-processing system together with the software programs written for interferogram analysis.

Application programs have been written by Becker (unpublished paper) to evaluate the interferograms along straight or curved lines represented by polygon lines. The program is run on the VT100 terminal, and the user is prompted for certain inputs such as reference points and fringe number assignment. After starting the program, a fringe pattern is digitized and frame- averaged to improve the signal-to-noise ratio. The user now inputs the location of two reference points to map the image-processor coordinates to the usercoordinate system by directing the system cursor to the appropriate locations and entering their positions in the user system. The reference points selected are usually the leading and trailing edges of the airfoil which are assigned the values 0.0 and 1.0 , respectively. The coordinates of the polygon line may be defined and written to a formatted VAX file before the evaluation, or may be interactively drawn into the image of the digitized interferogram using the joystick device. The analyses of this report, for instance, involved the evaluation of polygon lines just above the boundary layers of the upper and lower surface of the airfoils. This does not affect the results as the surface pressures are assumed to be constant across the boundary layer. Furthermore, there are no fringe patterns in the boundary-layer region that can be detected by the image processor. The system then displays back to the user the fringes that were detected along the polygon line as black and white segments (fringe segmentation). The obtained segmentation can then be accepted or corrected, and the user may then proceed to the numbering procedure.

During numbering, the cursor can be moved along the segmented polygon line positioning itself along the white segments. A reference fringe, whose density is known, is selected and assigned the value 0 . Subsequent fringes are numbered relative to the reference fringe in increments of 1 . The user determines the correct numbers to be assigned to each fringe by observing the entire flow pattern of the interferogram. If the surface pressure is known to increase to a certain point and then decrease, the user will correspondingly assign fringe numbers that indicate this increase and decrease. Depending on the polygon line being used, some fringes will have the same number as the fringe pattern bends in the opposite direction. Two different numbering modules may be used to set the fringe numbers. Only one number and the direction in which the fringe numbers increase have to be input by the user to number the entire line. During the numbering process, the black segments are overwritten by color to show the difference in the fringe order of two adjacent fringes. If the color indicated is green or blue, the fringe order increases or decreases by 
1 increment, respectively. If the color is red, the fringe order differs by more than 1 increment and the fringe order sequence is discontinuous. Black indicates that both fringes have the same number. The color coding is an ergonomic feature that makes numbering errors easier to detect. At all times one may go back to the segmentation procedure to start the numbering process again.

To be able to handle a wide range of fringe frequencies or increase the accuracy of the detection of fringes, the image-processor system is able to handle digitizations of several sections of one interferogram taken with different resolutions. Alternate lenses on the video camera are used to focus on appropriate sections. Each section is separately digitized until the entire polygon line has been evaluated. The application program is exited and the data from different portions of the interferogram are then merged into one set of data. The output will be the fringe numbers and location (fringe order function) along the polygon line being evaluated. The fringe order function is automatically output onto a VAX file and can be converted to interesting flow properties such as pressure coefficients, $C_{\mu}$, using a post-processing program.

\section{Ames 2- by 2-Foot Transonic Wind Tunnel}

The airfoils were tested in the Ames 2- by 2-Foot Transonic Wind Tunnel. The test facility is equipped with a holographic interferometer system which uses a Quanta Ray DCR-1 Nd:YAG laser as a light source. The laser is capable of producing a pulsed beam with rates between two and twenty per second at up to $80 \mathrm{~mJ}$ of energy in the green line $(0.532 \mu \mathrm{m})$. The hologram recording system is composed of transmitting and receiving components connected by two optical paths for the object and reference beams (ref. 2). A 4 by 5 -in film holder is used to hold the high-resolution holographic film plates for recording the information. This allows the storage of the data for later comparison and analysis outside the test facility. A reference hologram is recorded with no flow in the tunnel and a test hologram is recorded at the test condition. After processing, the reference and test holograms are reconstructed to an infinite-fringe interferogram (refs. 3,4). If the flow is two-dimensional, which is usually the case for airfoils, fringes on an infinite-fringe interferogram correspond to density contours in the flow, with a constant-density increment between fringes given by

$$
\rho_{x, y}-\rho_{r e f}=\frac{\lambda N}{L_{e f f} K_{C ;-D}}
$$




$$
\rho_{x, y}=\rho_{r e f}+(\text { constant }) N
$$

where $\rho_{x, y}$ and $\rho_{\text {ref }}$ are the densities of two adjacent fringes, $L_{e f f}$ is the effective optical path length including the effect of the sidewall boundary layers, $\lambda$ is the laser wavelength, $K_{G-D}$ is the Gladstone-Dale constant and $\mathrm{N}$ is the fringe order number (ref. 5). In equation (2), the known test constants are replaced by a single constant as indicated. As can be seen in equation (2), the reference fringe of value $N=0$, will have the reference density at that position and must be known for this fringe. The density can be determined in several ways. If there is a region of undisturbed flow in the field of view, the free-stream density can be used. Unfortunately, this is not generally the case. Instead, a surfacepressure measurement can be converted to density by using the total temperature and total pressure if isentropic flow conditions are assumed. If the free-stream density is used as the reference density, along with its corresponding fringe of $\mathrm{N}=0$, equation (2) can be written as follows:

$$
\rho_{x, y}=\rho_{\infty}+(\text { constant }) N
$$

The constant values in the experiments conducted in the Ames 2- by 2-Foot Transonic Wind Tunnel facility to obtain the interferograms are given in table 1 . Note that a Ruby laser was used for the interferograms taken of the symmetric airfoil whereas the Nd:YAG laser was used for the supercritical and circulation-control airfoils. 
TABLE 1. - TEST CONSTANTS

\begin{tabular}{|c|l|l|l|l|l|}
\hline $\begin{array}{l}\text { INTERFE- } \\
\text { ROGR AM }\end{array}$ & AIRFOIL & LASER & $\begin{array}{c}\lambda, \\
\mu m\end{array}$ & $\begin{array}{c}K_{G_{i}-D}, \\
\mathrm{~cm}^{3} / g\end{array}$ & $\begin{array}{c}L_{\text {eff }}, \\
\mathrm{mm}\end{array}$ \\
\hline I & $64 \mathrm{A010}$ & RUBY & 0.6934 & 0.225 & 609.6 \\
II & $64 \mathrm{A010}$ & RUBY & 0.6934 & 0.225 & 609.6 \\
III & DSMA671 & Nd:YAG & 0.5320 & 0.226 & 609.6 \\
IV & DSMA671 & Nd:YAG & 0.5320 & 0.226 & 609.6 \\
V & CIRCON & Nd:YAG & 0.5320 & 0.226 & 609.6 \\
VI & CIRCON & Nd:YAG & 0.5320 & 0.226 & 609.6 \\
\hline
\end{tabular}

The substitution of these values into equation (2) and the use of the test section width of $2 \mathrm{ft}$ for $L_{e f f}$, since boundary layer correction is less than $2 \%$ (i.e., $L=L_{e f f}$ ), gives

$$
\rho_{x, y}=\rho_{r e f}+5.06 \times 10^{-3} N
$$

for the Ruby laser and

$$
\rho_{x, y}=\rho_{r e f}+3.88 \times 10^{-3} N
$$

for the Nd:YAG laser.

The only variable left is the fringe order number, $\mathrm{N}$, which is obtained from the digitized interferograms. Once the densities are known, other aerodynamic information can be obtained. For example, if isentropic flow conditions are assumed, the pressure coefficients are derived as follows

$$
C_{p}=\frac{2}{\kappa M_{\infty}^{2}}\left[\left(\frac{\rho_{x, y}}{\rho_{r e f}}\right)^{\kappa}-1\right]
$$




\section{Models}

The airfoils for which holographic interferograms were obtained and evaluated on the image-processing system included a symmetrical airfoil (64A010) (ref. 6) a supercritical airfoil (DSMA671) (ref. 7) and a circulation-control airfoil (CIRCON) (ref. 8). Interferograms were obtained in tests conducted in the Ames 2- by 2-Foot Transonic Wind Tunnel facility which is a closed-return, variable-density tunnel with baffled, slotted upper and lower walls for transonic testing.

The 64A010 airfoil is a geometrically simple, symmetrical airfoil section. The model was a 6-in. chord airfoil which spanned the entire test section. Tunnel conditions were fixed at a free-stream Mach number of 0.8 and a Reynolds number of $2 \times 10^{6}$.

The supercritical airfoil tested was the DSMA671 airfoil of the DSMA series, which is characterized by the relatively flat upper surface and cambered trailing edge (see figs. 6 and 8 ).

The CIRCON is a CIRculation CONtrol airfoil which utilizes the Coanda effect at the trailing edge for lift control. The Coanda effect occurs when a high-velocity fluid jet is ejected tangentially from a surface slot. The jet attaches itself to the surface because of the reduced static pressure it produces. The airfoil tested was elliptic with a thickness ratio of $21 \%$, a camber of $3 \%$, and a 6 -in. chord. The blowing slot was located at $96.2 \%$ of the chord.

Six interferograms, two for each airfoil model, were evaluated on the image-processor system. Table 2 shows the test conditions for which the interferograms were obtained.

TABLE 2. - TEST CONDITIONS

\begin{tabular}{|l|l|l|l|}
\hline $\begin{array}{l}\text { INTERFE- } \\
\text { ROGRAM }\end{array}$ & AIRFOIL & MACH NO. & $\begin{array}{c}\text { ANGLE OF } \\
\text { ATTACK, } \\
\alpha, \mathrm{deg}\end{array}$ \\
\hline I & $64 \mathrm{~A} 010$ & 0.80 & 0.00 \\
II & $64 \mathrm{~A} 010$ & 0.80 & 6.50 \\
III & DSMA671 & 0.72 & 4.32 \\
IV & DSMA671 & 0.72 & 6.10 \\
V & CIRCON & 0.50 & 0.00 \\
VI & CIRCON & 0.65 & 0.00 \\
\hline
\end{tabular}




\section{RESULTS}

Fringe-order functions were obtained for the upper and lower surfaces of interferograms I through VI of table 2 using the image-processor system. Equation (8) was then used to obtain $C_{k}$ data. The results are plotted in figures 3-13 and compared with data obtained by other methods. The latter included experimental data from pressure orifices on the airfoils, providing a direct reading of the surface pressures, and the manual technique of counting the fringes of an infinite-fringe interferogram by hand and then applying the previously discussed equations. All three methods are compared to see how the imageprocessor system agrees with experimental and hand-evaluated data. If the results prove to be in good agreement with the pressure-transducer measurements, the image processor is justified as the best method to use in such evaluations because it would be the most efficient, easiest, and fastest method.

\section{DISCUSSION}

The interferograms were digitized on the De Anza image processor. The actual digitization process takes only a few seconds. However, the total time required to evaluate an interferogram averages out to be about $30 \mathrm{~min}$. This includes setting up the equipment, running the application program, and running some post-processing programs. If everything is set up, the time required to evaluate an interferogram would be about 15 min. This includes running the application program from start to finish, and inputting of reference points, creating polygon lines, numbering fringes, and changing camera lens, when required.

The interferograms were chosen to test the capabilities of the image-processor system for a wide variety of fringe patterns. The different geometries of the airfoils together with the different test conditions resulted in flows with strong and weak shocks, separated flows, and flows with strong pressure gradients, all of which created unique fringe patterns. The general technique for running the application program was the same for all interferograms; however, the creation of polygon lines was unique to each case, depending on the fringe patterns.

Figure 2 shows the infinite-fringe interferogram for the $64 \mathrm{~A} 010$ symmetrical airfoil at Mach 0.8 and a $0^{\circ}$ angle of attack. This flow has a weak shock at $\mathrm{X} / \mathrm{C}=0.45$ and $\mathrm{a}$ symmetric fringe pattern. The fringes are very distinct and clear for this interferogram, making it easy to evaluate on the image processor. The only difficulty involved the leading edge where blurring of fringes occured. The polygon lines were created at the edge of the thin boundary layer for the upper and lower surfaces, and the image processor picked up all of the fringes, except for the leading-edge area, during the segmentation process. The method for selecting the reference fringe, $\mathbf{N}=0$, for this interferogram was to choose it at free-stream conditions rather than to use a known pressure measurement. That is, this fringe was observed to go to a region far away from the airfoil where it can be assumed to be at free stream. This fringe is not bending around the local density field but rather extends into the undisturbed free-stream region of the wind tunnel. The fringe was traced down to the local airfoil surface where the polygon line was drawn, which happened to be at the 
airfoil $\mathrm{X} / \mathrm{C}=0.73$ location and assigned the number $\mathrm{N}=0$. This made it possible to use equation (3) to obtain the remaining density field as the remaining fringes were numbered relative to $N=0$. The video camera lens was changed to enlarge the blurred region near the leading edge of the airfoil, but very few additional fringes were detected. Instead, the fringe numbers were estimated in this area. This was done by visually extrapolating known fringes from a clear region into the blurred region and then assigning the numbers to the few fringes detected in the blurred region.

Figure 3 shows the corresponding surface pressures for interferogram I. As can be seen, both methods show the same $C_{p}$, values for upper and lower surfaces as expected, with a maximum suction at about -0.68 at an $\mathrm{X} / \mathrm{C}$ of 0.45 . Discrepancies can be noted at the leading and trailing edges of the airfoil. Estimation of fringe numbers in the leading-edge area resulted in error in the data. However, for the region between $0.1 \leq X / C \leq 0.9$, the results are very good to within $1 \%$ of the pressure data. The region near the trailing edge also shows a discrepancy which was probable due to the subjective choice of the reference fringe where human error could have caused the reference fringe to be two or three fringes from the true free-stream conditions. Another source of error may have been in selecting the second reference point $(1,0)$, as the end of the airfoil was not clearly visible in the interferogram. Better results would have been obtained for this case if the quality of the interferogram had been better.

Figure 4 shows the infinite-fringe interferogram for the same $64 \mathrm{~A} 010$ symmetrical airfoil at the same Mach number but with the angle of attack increased to $6.5^{\circ}$. A strong shock forms at about $\mathrm{X} / \mathrm{C}=0.4$ and the flow becomes separated at this point. Because of the presence of the shock on the upper surface and the large number of fringes in the leading edge of the airfoil, the interferogram was evaluated in four sections, two before and two after the shock. For the region, $0.0 \leq X / C \leq 0.4$, the polygon line for the upper surface was drawn close to the edge of the boundary layer. The fringe pattern was relatively flat in this region so that the same fringe number was assigned in several locations with fluctuations between two or three numbers. The region of separated flow, $0.4 \leq X / C \leq 1.0$, can be seen very clearly after the shock. For the purposes of evaluating the interferogram, this region can be considered to be a thick boundary layer. It is a subjective choice as to where to draw the polygon line, but it was selected just outside the thick boundary layer before the fringes begin to bend drastically. The pressure was assumed to be constant through the boundary-layer region and normal to the local airfoil surface. $\mathrm{N}=0$, the reference fringe, was chosen from a known pressure measurement as was the case for the remaining interferograms. The remaining fringes were assigned numbers relative to this fringe. A fringe that was observed to go around the shock from the first section was traced into the second section and assigned the same number; the remaining fringes were then numbered. The lower surface of the airfoil had a clear fringe pattern and was numbered in the same manner as interferogram I with a similar polygon line.

Figure 5 shows the resulting surface pressures for interferogram 1I. The presence of the shock is clearly seen in the graph as the $C_{p}$ drops from -1.2 to -0.4 at an $\mathrm{X} / \mathrm{C}$ of 0.4 . It would be advantageous to have four direct pressure measurements for future interferogram analysis of airfoils with shocks to obtain known pressures before and after the shock, two transducers for each surface. Blurring of fringes causes some error in the leading edge 
for the lower surface region between $0.0 \leq X / C \leq 0.15$. In the remaining trouble-free region of the interferogram, the results agree with the transducer measurements to within 1\%. Figure 5 also shows the quantitative data obtained by manual techniques. This involved manually counting each fringe on the interferogram starting from a reference fringe location. These data were input into a desk top computer for which software had been written to reduce the information.

Figure 6 is the interferogram for the DSMA671 supercritical airfoil at a Mach number of 0.72 and angle of attack of $4.32^{\circ}$. This interferogram has a high number of fringes in the leading edge area up to the shock, $\mathrm{X} / \mathrm{C}=0.37$, and a clearly defined boundary layer and wake after the shock for both upper and lower surfaces. The interferogram was also evaluated in four sections, two before and two after the shock. The polygon line was drawn just outside the outer edge of the boundary layer and wake. Toward the trailing edge, it can be seen that the fringes begin to bend slightly where the outer edge of the boundary layer is located; the polygon line was drawn below the bend but above the boundary layer. This was done to obtain the actual end location of the fringes as they touched the boundary layer. The image processor detected the clear fringe pattern and the fringes were numbered relative to the reference fringe. In the upper surface, the fringe pattern gets flat for about $0.37 \leq X / C \leq 0.57$ so that only about seven fringes are located in this region; however, they remain clear and are easily numbered. The fringes are also easily numbered for $0.57 \leq X / C \leq 1.0$. It was more difficult to number the region before the shock. For the region $0.0 \leq X / C \leq 0.1$, the original interferogram has too many fringes for fringe detection by the image processor and fringe numbers have to be estimated along the polygon line. However, the region from $0.1 \leq X / C \leq 0.37$ can be numbered more easily. A fringe was traced around the shock from the aft section and was assigned the same number in the forward region, the same technique used in interferogram II. The lower surface was a little easier to evaluate since there was no shock present although the leading edge area also had a high density of fringes. The polygon lines were drawn as in the upper surface.

Figure 7 shows the surface pressures obtained for interferogram III. The discrepancies occur mostly before the shock where the high fringe density was located, resulting in error for the lower surface in particular. In regions aft of the shock, the agreement is better because of the clear fringe patterns and well-defined boundary layer. The agreement with pressure measurements after the shock is within $1 \%$.

Figure 8 is the interferogram for the DSMA671 supercritical airfoil at the same Mach number, but with angle of attack increased to $6.1^{\circ}$. The interferogram is very similar to interferogram III, with the same problem of too many fringes near the leading edge, causing error in the lower surface. It was evaluated in a similar manner.

Figure 9 shows the corresponding surface pressures for interferogram IV. As can be seen, the results are very similar to the previous case and are in good agreement with the pressure measurements to within $1 \%$. The poor agreement near the leading edge for the lower surface, resulting from the high fringe concentration caused by large density gradients, can be seen for the region $0.0 \leq X / C \leq 0.1$.

Figure 10 is the interferogram for the CIRCON airfoil. As can be seen in the interferogram, the fringes are symmetric about $\mathrm{X} / \mathrm{C}=0.5$ and flatten out near the boundary layer of the upper surface. Blurring due to a high concentration of fringes made it difficult 
to number in the region $0.0 \leq X / C \leq 0.1$ for the upper surface. Near the trailing edge, the high-pressure gradients of the Coanda jet affected the fringe patterns and the quality of the interferogram in the region $0.8 \leq X / C \leq 1.0$. This problem was overcome by evaluating the interferogram in five sections. A very thin boundary layer can be assumed for this type of airfoil, and consequently the polygon lines were drawn as close as possible to the airfoil surface. The lower surface had a large model support obstructing the fringes, but because the fringe pattern was observed to be very flat, the polygon line was drawn close to the airfoil and assumed to be constant with the fringe numbers extrapolated from the unobstructed lower surface region. Because of the flat fringe pattern, only about 15 fringes had to be numbered for the lower surface, and most of these were located near the trailing edge.

Figure 11 shows the surface pressures obtained from interferogram V. The results were very good to within $1 \%$ of pressure measurements since the evaluation was performed in five sections. However, partial model support blockage on the lower surface required that some fringe numbers had to be estimated as a large section was assumed to be constant. The correct symmetry of the CIRCON airfoil can be discerned about $\mathrm{X} / \mathrm{C}=0.5$ with a maximum $C_{p}$ value of -1.0 for the upper surface, and a relatively constant $C_{p}$ value of about -0.1 for $0.2 \leq X / C \leq 0.8$ for the lower surface.

Figure 12 has the interferogram for the CIRCON airfoil at Mach 0.65 and an angle of attack of $0^{\circ}$. The interferogram is similar to interferogram $\mathrm{V}$, with a disturbed blowing pressure region near the trailing edge and a large obstructed region in the leading edge as well as in the lower surface. The effects can be seen as a blurring of fringes in the leading edge, and a distortion of the fringe pattern in the trailing edge. The lower surface blockage shows up as a black region in the interferogram. The polygon lines were drawn as in interferogram $\mathrm{V}$.

Figure 13 shows the resulting surface pressures of interferogram VI. As with interferogram V, there was a high concentration of fringes in the leading edge which caused blurring in the upper surface. The discrepancies can be seen in the region $0.0 \leq X / C \leq 0.2$, although the results are just as good as the manual techniques data. In the region $0.35 \leq X / C \leq 0.6$, the image processor data is better than the manual technique data. While in the trailing edge area, image processor and manual technique data are in agreement but both are off from pressure measurements due to the Coanda jet effects. The jagged nature of the data for the lower surface is due to model support blockage. As with interferogram $\mathrm{V}$, the polygon line was assumed to be constant in the blocked area with fringe numbers estimated in this region. The interferogram was evaluated in the same way as interferogram $\mathrm{V}$ and the results are to the same accuracy. 


\section{CONCLUSIONS}

Interferograms can be digitized and evaluated using a semiautomatic image processor. The results with image processing techniques depend heavily on the quality of the original image. Care must taken to obtain clear fringe patterns, especially in the leading and trailing edges of the airfoil where many fringes converge in a relatively small area and blurring of patterns can occur. Image-enhancement techniques will not completely solve this problem as the entire image-processing procedures provide opportunity for image degradation. It was shown, however, that in regions where fringe patterns are very sharp and clear, the evaluation of interferograms proceeds with ease and yields very good results. The problems associated with fringe blurring and distortion can be handled by increasing the number of sections to be evaluated thereby effectively increasing the resolution. It was also found that a wide variety of fringe patterns can be evaluated on the image processor. Interferograms with shocks, separated flows, and high-pressure regions can be handled provided care is taken in selecting the polygon lines to be evaluated and correct assumptions are made. Perhaps additional software may be developed in the future to aid in fringe enhancement and detection. Fringes that are very clear in one region of the flow, for instance, may be extrapolated to a region that is blurred where they can be detected by the image processor. 


\section{REFERENCES}

1. Becker, F.; and Yu, Y.H.: Digital Fringe Reduction Techniques Applied to the Measurement of Three Dimensional Transonic Flow Fields. Opt. Eng., vol. 24, no. 3, May/June 1985, pp. 429-434.

2. Vest, C.M.: Holographic Interferometry. Wiley \& Sons, Inc., 1978.

3. Trollinger, J.D.: Laser Instrumentation for Flow Field Diagnostics. AGARD AG186, 1974.

4. Lee, G.: Application of Holography to Flow Visualizations. NASA TM 84325, 1984.

5. Spaid, F.W.; and Bachalo, W.D.: Experiments on the Flow About a Supercritical Airfoil Including Holographic Interferometry. J. Aircraft, vol. 18, no. 4, Apr. 1981, pp. 287-294.

6. Johnson, D.A.; and Bachalo W.D.: Transonic Flow Past a Symmetrical Airfoil Inviscid and Turbulent Flow Properties. AIAA J., vol. 18, no. 1, Jan. 1980, pp. 16-24.

7. Bachalo, W.D.: Measurements of Supercritical Airfoil Flow Fields Using Interferometry. Aerometrics, Inc., Mountain View, CA, Mar. 22, 1982.

8. Bachalo, W.D.: An Experimental Investigation of Circulation Control Flow Fields Using Holographic Interferometry. NASA CR 166482, Oct. 1982. 
HARDWARE COMPONENTS

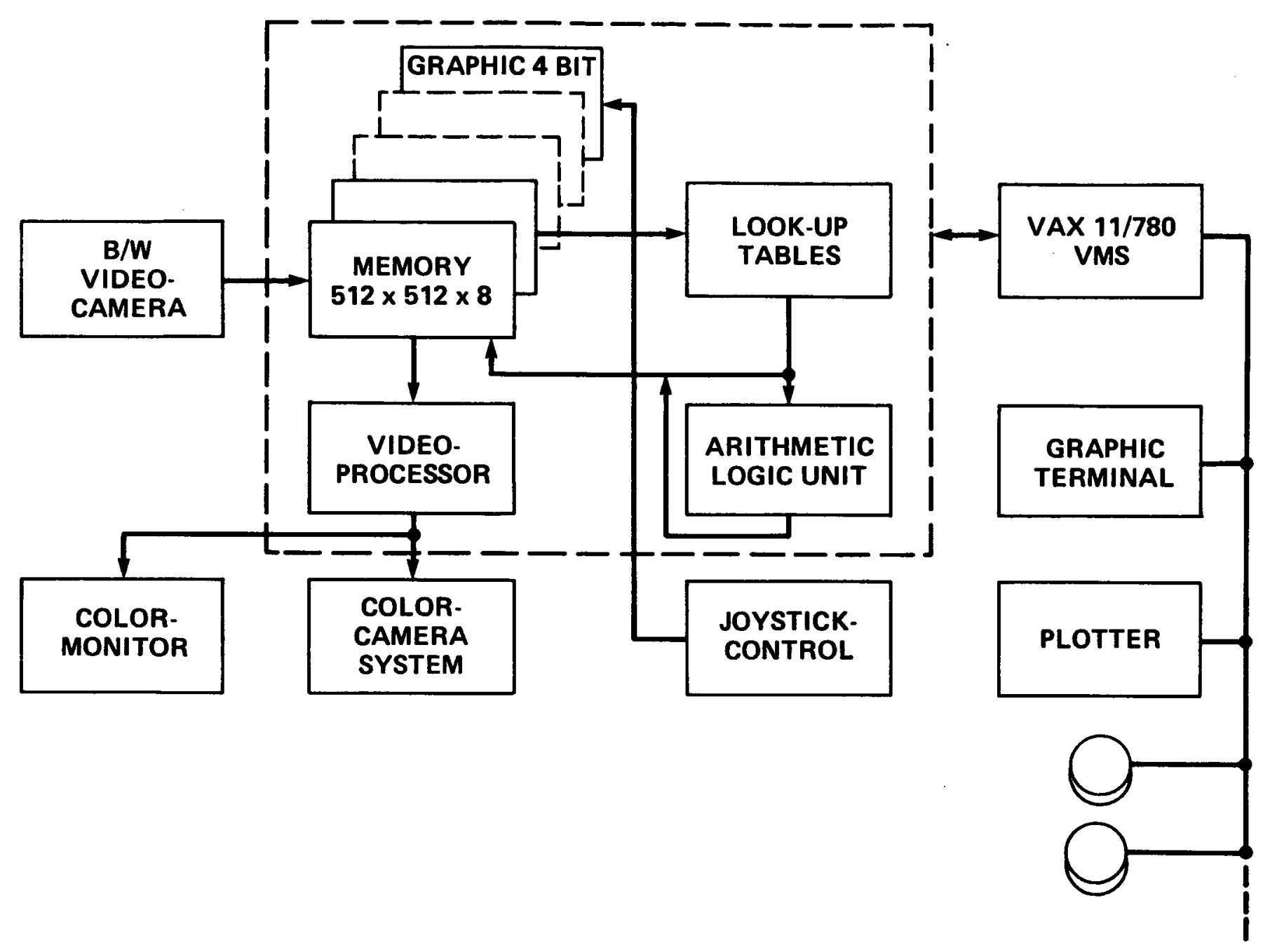

Figure 1.- De Anza image-processing system. 


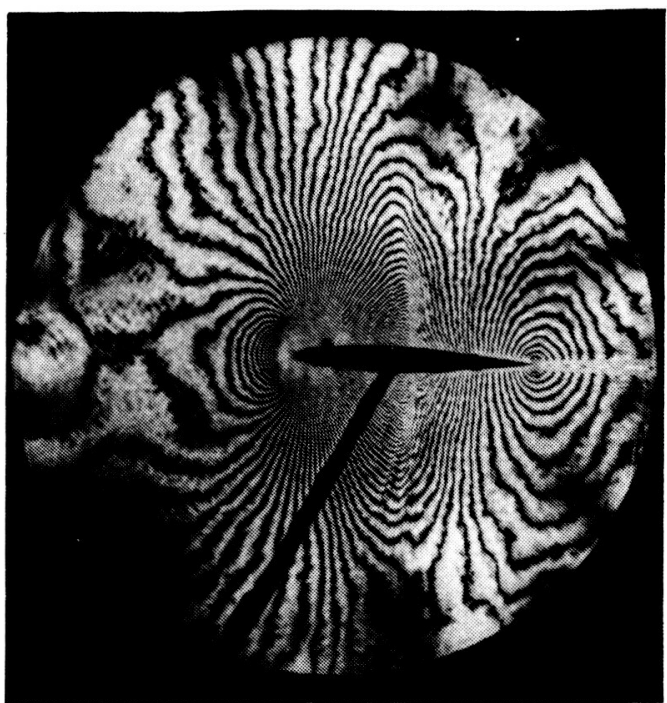

Or POCA GATI

Figure 2.- Interferogram I ( $64 \mathrm{~A} 010$ airfoil, $\mathrm{M}=0.8, \mathrm{~A}=0.0$ ).

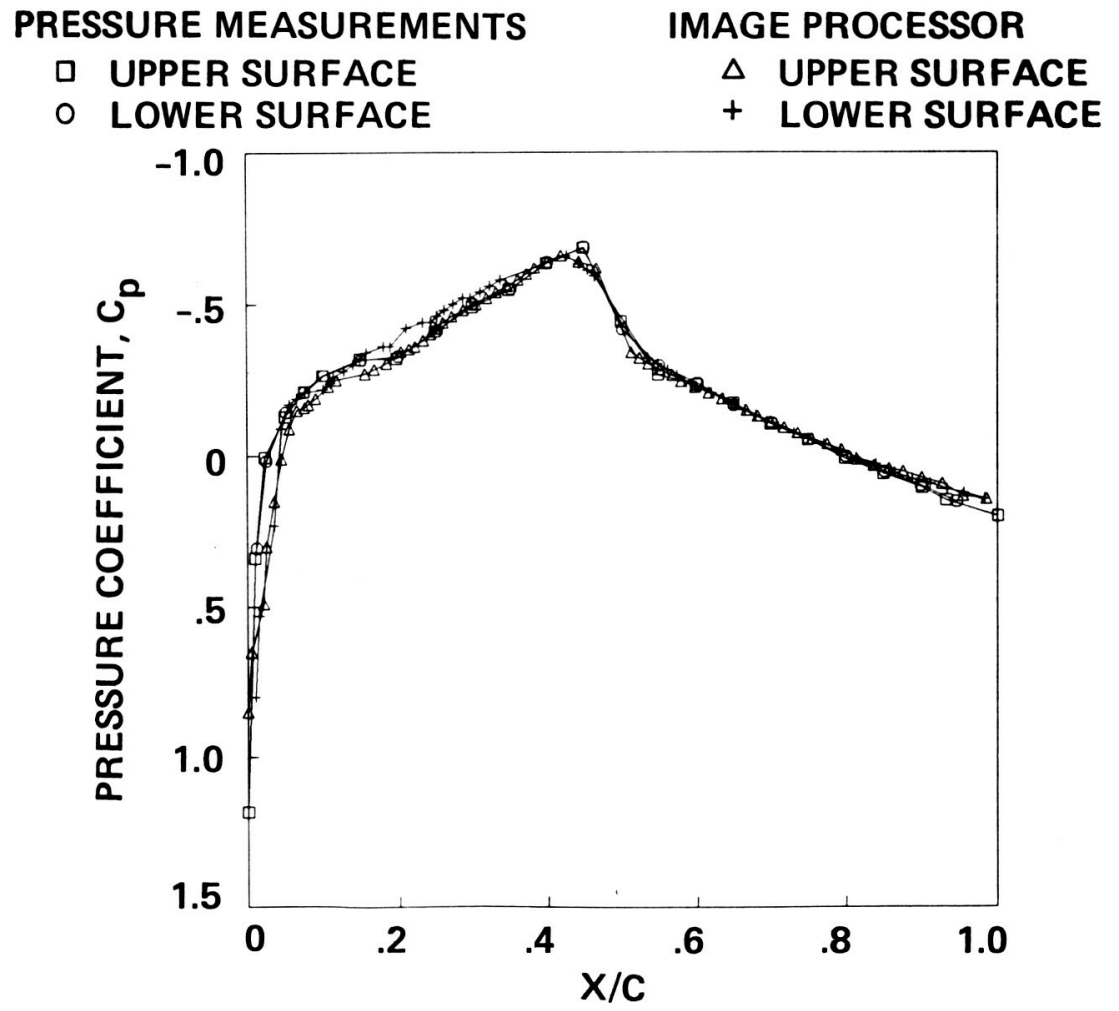

Figure 3.- Surface pressures for $64 \mathrm{~A} 010$ airfoil $(\mathrm{M}=0.8, \mathrm{~A}=0.0)$. 


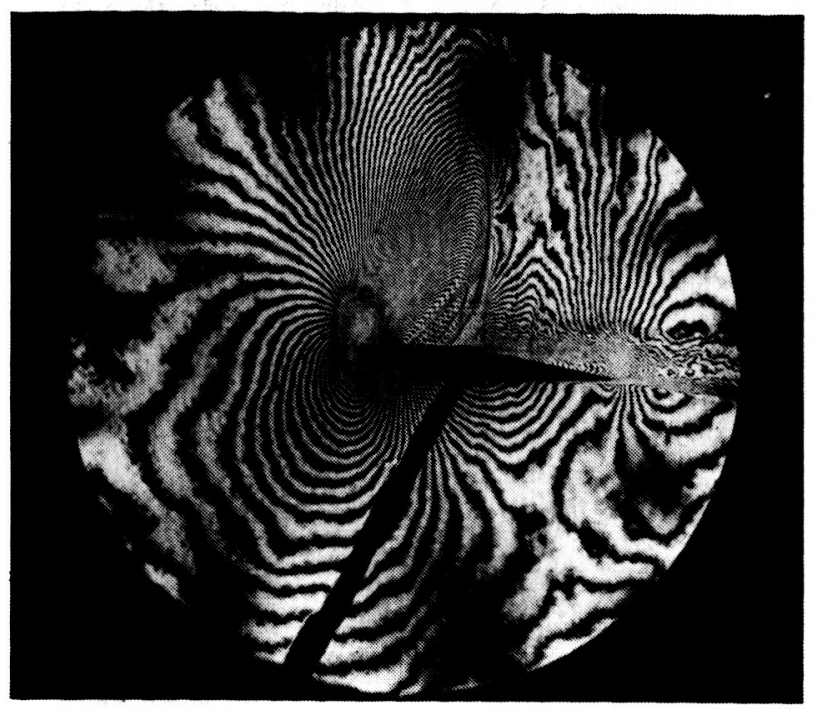

Figure 4. - Interferogram II (64A010 airfoil, $\mathrm{M}=0.8, \mathrm{~A}=6.5$ ).

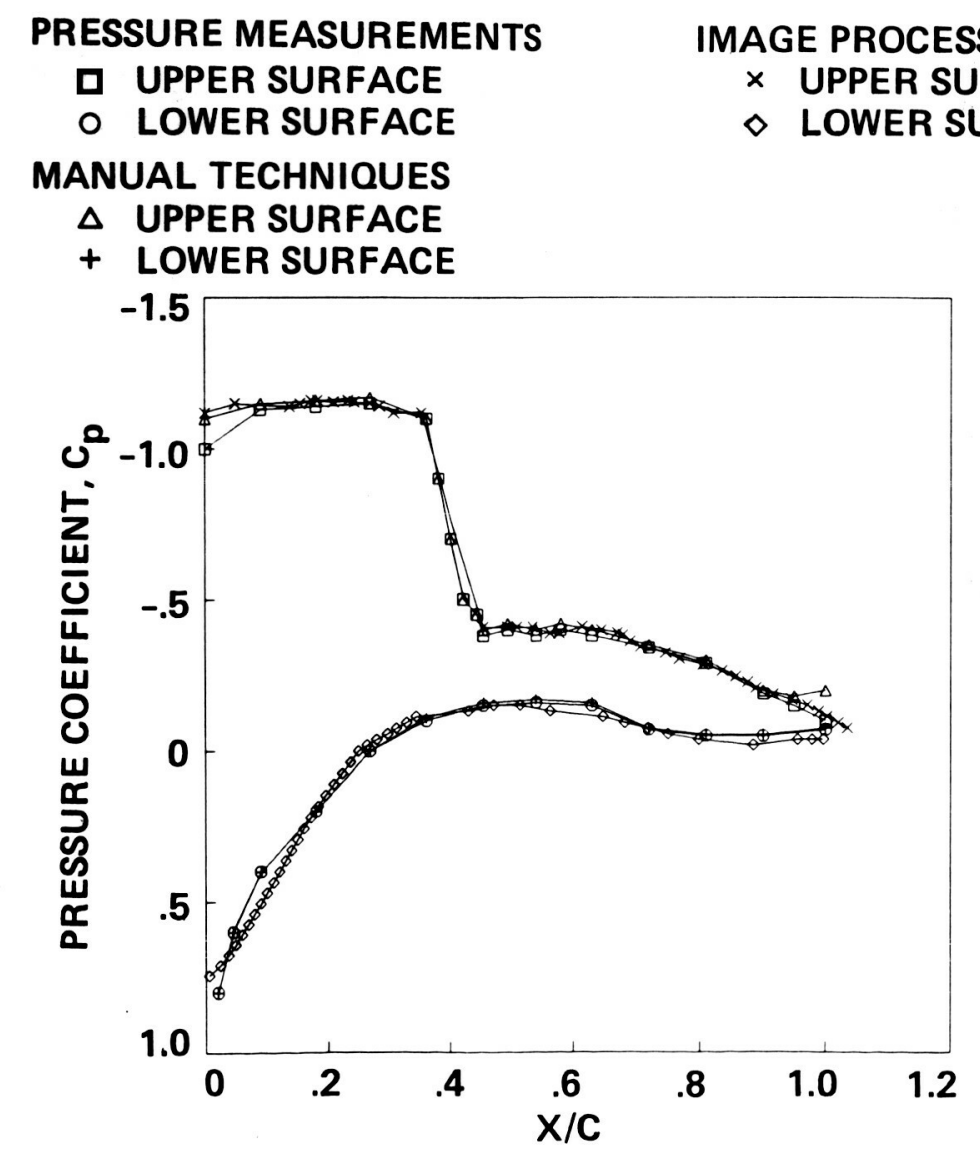

Figure 5.- Surface pressures for $64 \mathrm{~A} 010$ airfoil $(M=0.8, A=6.5)$. 


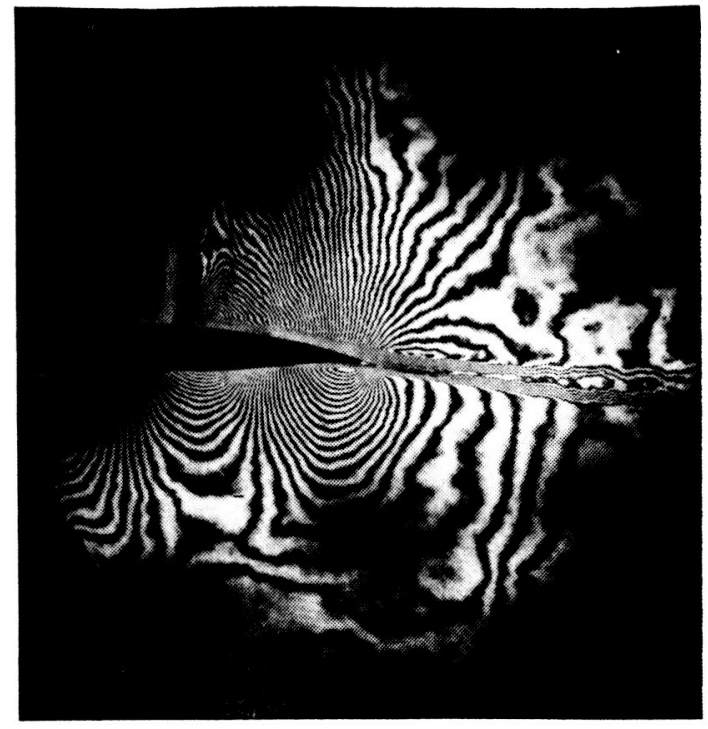

Figure 6. - Interferogram III (DSMA671 airfoil, $\mathrm{M}=0.72, \mathrm{~A}=4.32$ ).
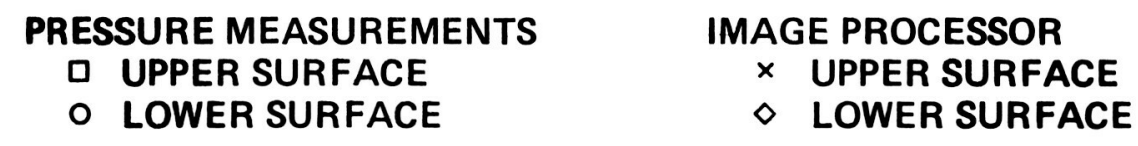

MANUAL TECHNIQUES

$\triangle$ UPPER SURFACE

+ LOWER SURFACE

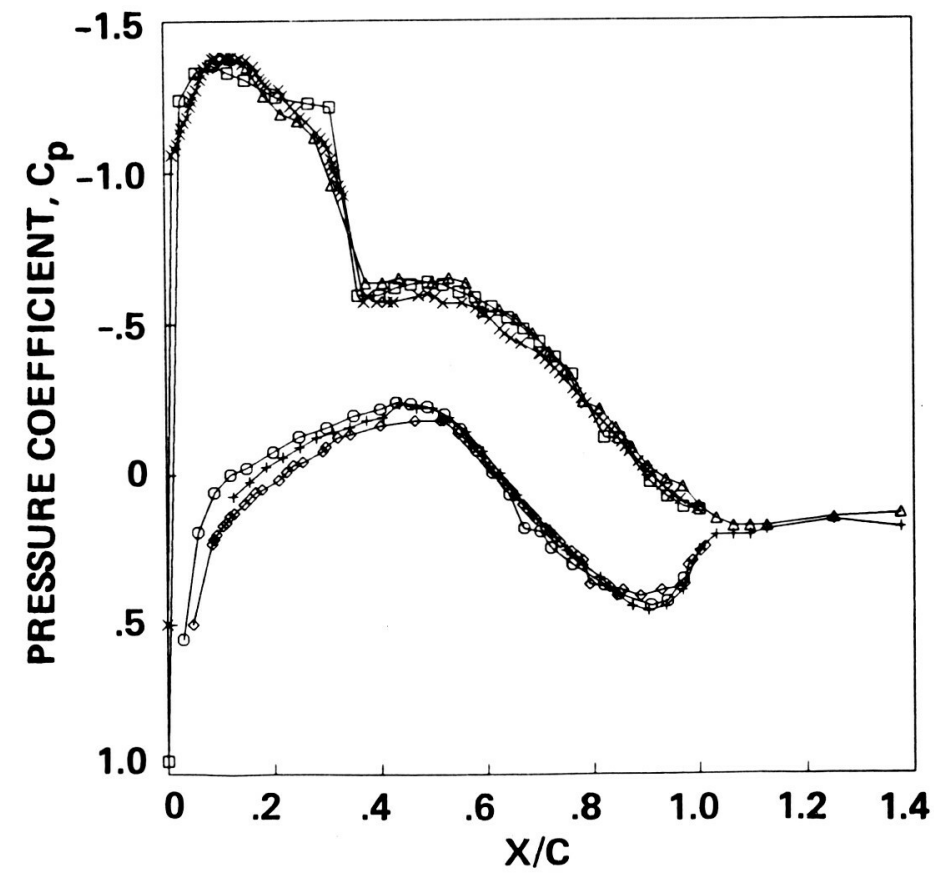

Figure 7.- Surface pressures for DSMA671 airfoil $(M=0.72, A=4.32)$. 


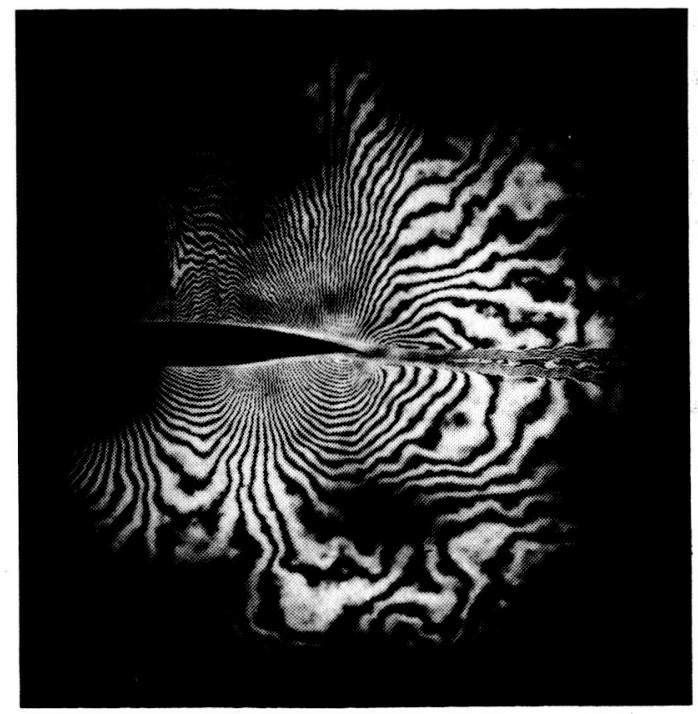

Figure 8.- Interferogram IV (DSMA671 airfoil, $\mathrm{M}=0.72, \mathrm{~A}=6.1$ ).

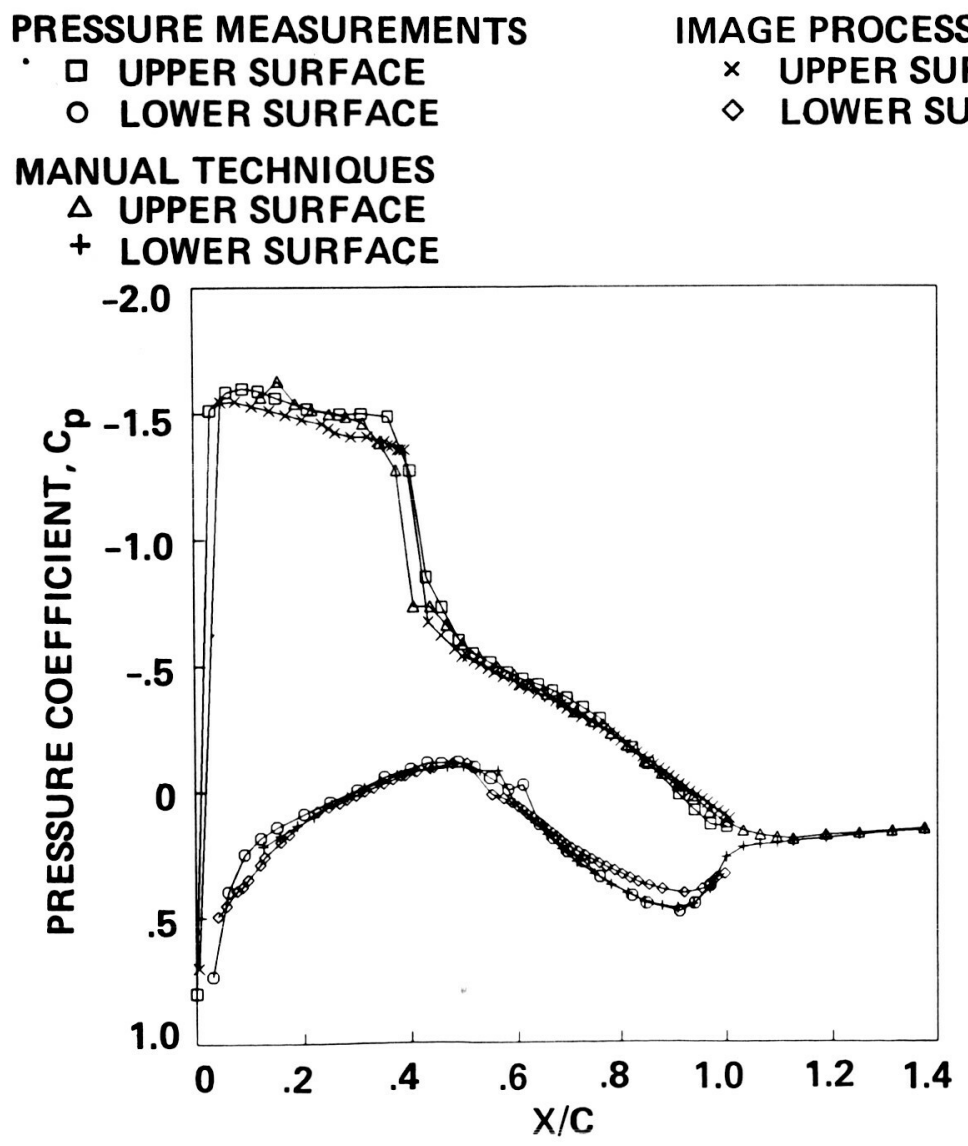

Figure 9.- Surface pressures for DSMA671 airfoil $(M=0.72, A=6.1)$. 


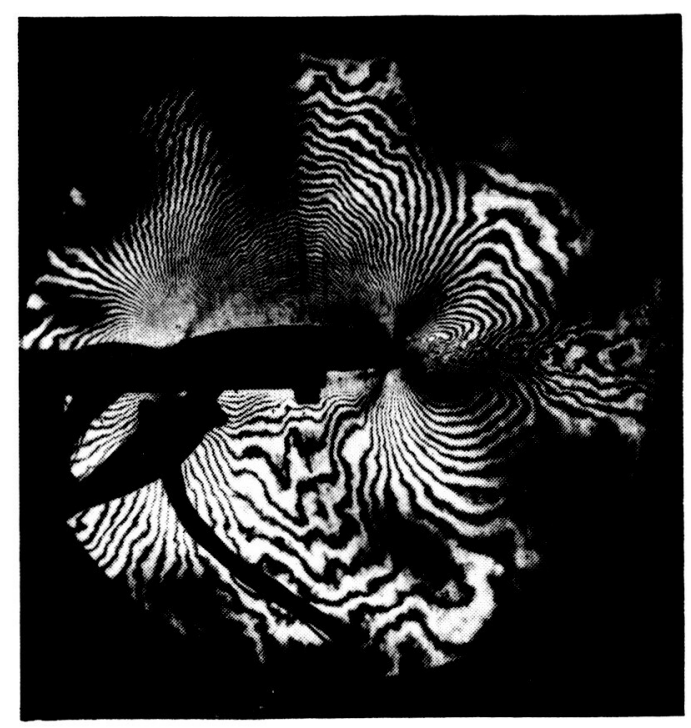

Figure 10.- Interferogram V (CIRCON airfoil, $\mathrm{M}=0.5, \mathrm{~A}=0.0)$.

PRESSURE MEASUREMENTS

- D UPPER SURFACE

- LOWER SURFACE

MANUAL TECHNIQUES

$\triangle$ UPPER SURFACE

+ LOWER SURFACE

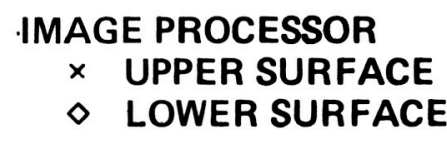

IMAGE PROCESSOR

$\diamond$ LOWER SURFACE

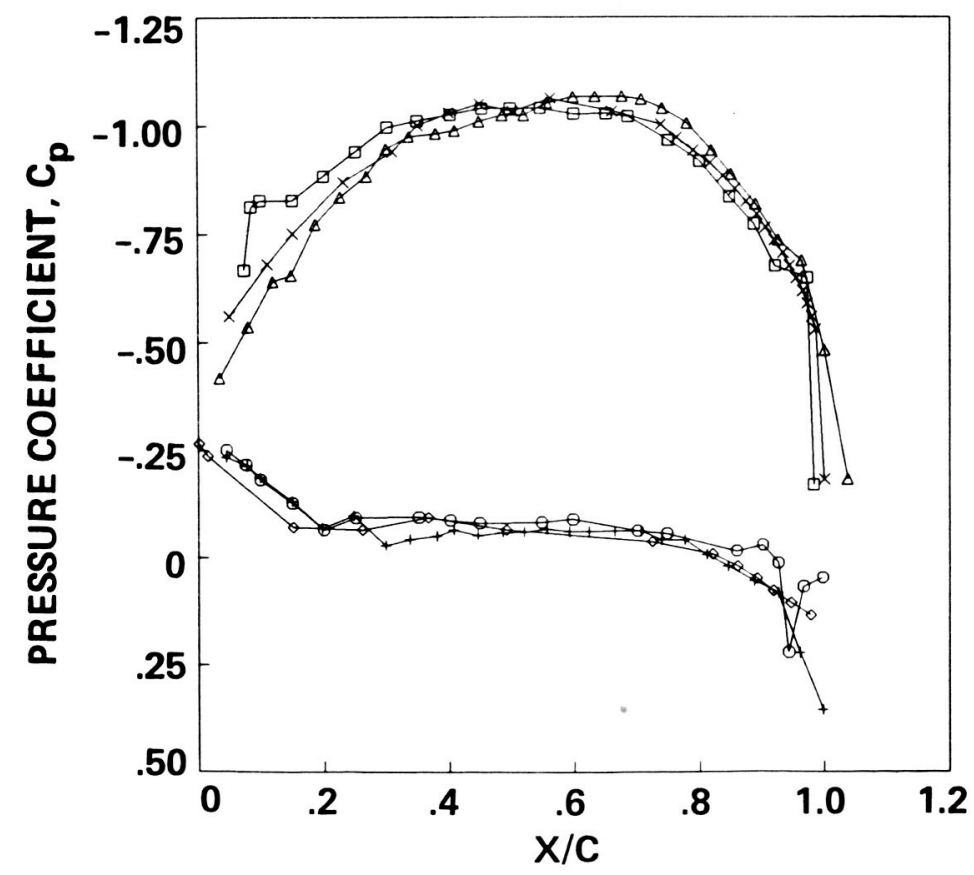

Figure 11.- Surface pressures for CIRCON airfoil $(\mathrm{M}=0.5, \mathrm{~A}=0.0)$. 


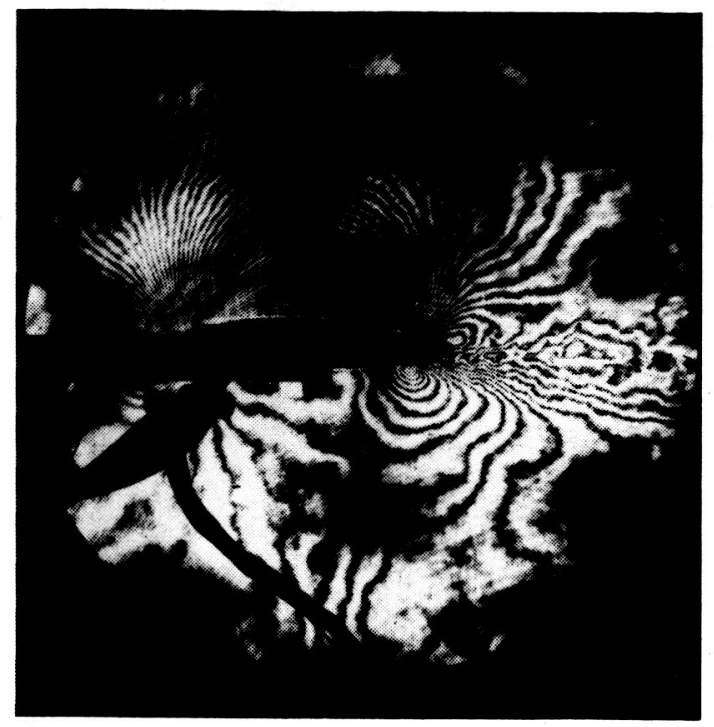

ORIGINAL PRES IS

OF. POOR QUALITY

Figure 12.- Interferogram VI (CIRCON airfoil, $\mathrm{M}=0.65, \mathrm{~A}=0.0)$.

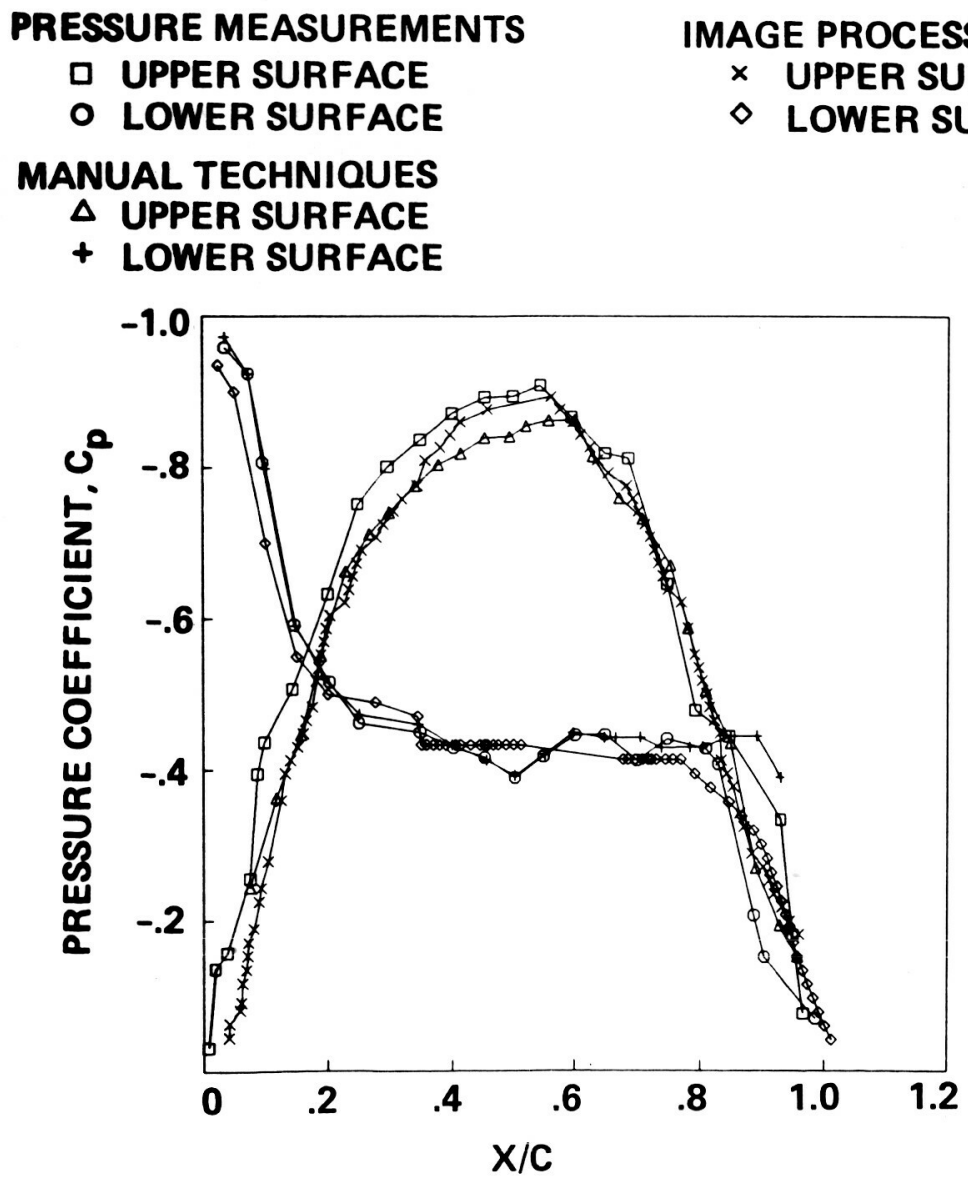

Figure 13.- Surface pressures for CIRCON airfoil $(M=0.65, A=0.0)$. 


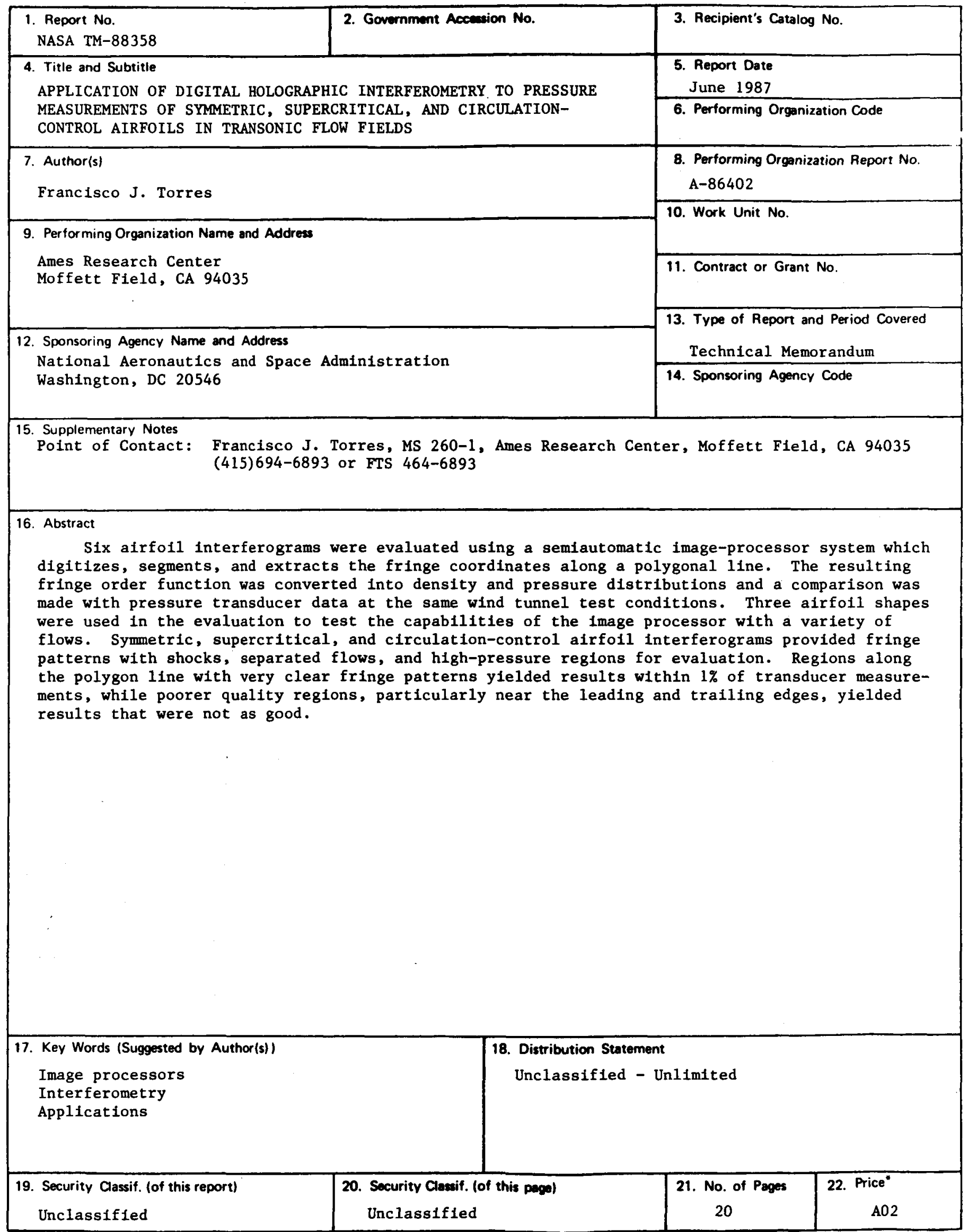

